Relations industrielles

Industrial Relations

\title{
La Chambre de Commerce du Canada, la liberté d'entreprise, les relations patronales-ouvrières
}

Volume 17, numéro 3, juillet 1962

URI : https://id.erudit.org/iderudit/1021581ar

DOI : https://doi.org/10.7202/1021581ar

Aller au sommaire du numéro

Éditeur(s)

Département des relations industrielles de l’Université Laval

ISSN

0034-379X (imprimé)

1703-8138 (numérique)

Découvrir la revue

Citer ce document

(1962). La Chambre de Commerce du Canada, la liberté d'entreprise, les relations patronales-ouvrières. Relations industrielles / Industrial Relations, 17(3), 337-345. https://doi.org/10.7202/1021581ar
Résumé de l'article

Le 23 novembre 1961, la Chambre de Commerce du Canada présentait au Cabinet fédéral un important mémoire. Nous en extrayons certaines parties qui ont trait à la philosophie et aux problèmes des relations patronales-ouvrières.
Tous droits réservés ( Département des relations industrielles de l’Université Laval, 1962
Ce document est protégé par la loi sur le droit d'auteur. L’utilisation des services d'Érudit (y compris la reproduction) est assujettie à sa politique d'utilisation que vous pouvez consulter en ligne.

https://apropos.erudit.org/fr/usagers/politique-dutilisation/ 


\title{
INFORMATIONS
}

\section{LA ChAMBre DE COMMERCE DU CANADA, LA LIBERTÉ D’ENTREPRISE, LES RELATIONS PATRONALES-OUVRIËRES}

\author{
Le 23 novembre 1961, la Chambre de Commerce du Canada présentait \\ au Cabinet fédéral un important mémoire. Nous en extrayons certaines \\ parties qui ont trait da la philosophie et aux problèmes des relations \\ patronales-ouvrières.
}

'_IBERTÉ D'ENTREPRISE

\section{Régime économique du Canada}

La Chambre de Commerce du Canada croit au régime de libre entrcprise, fondé sur la liberté individuelle et l'initiative privée, et l'appuie totalement.

La Chambre de Commerce du Canada professe que l'un des buts premiers de toutes nos activités publiques devrait être la préservation de la liberté individuelle. Dans un climat de liberté le consommateur est libre de décider de la manière dont il emploiera son revenu. La somme de ces décisions, de ces choix individuels, offre à la fois un stimulant et un frein aux facteurs de production. Les producteurs et les fournisseurs doivent être libres de se faire concurrence mutuellement dans la création de produits nouveaux et dans l'amélioration de ceux déjà sur le marché. Sous un régime de libre concurrence seuls les produits de bonne qualité trouvent preneurs et les producteurs qui négligent la qualité ou qui s'obstinent à fabriquer de la marchandise désuète sont voués à l'insuccès. La concurrence a pour effet d'amener l'amélioration de la production pour le plus grand bien des consommateurs. De là l'origine des expressions «Liberté d'entreprise» et «régime de libre concurrence ».

Le Canada ne peut être prospère qu'à la condition que tous ses citoyens jouissent du stimulant qu'est la rémunération pour les risques encourus, l'énergie et l'initiative déployées, ainsi que du fruit de leur économie et de leur prévoyance.

La Chambre croit que son premier souci est d'étayer le système de libre entreprise, et croit que dans les cadres de la liberté individuelle certaines restrictions sont justifiées dans l'intérêt de l'ordre et de la justice. Toutefois, l'usage abusif de pouvoirs discrétionnaires par les gouvernements, souvent inspirés par des concepts collectivistes, sapent les initiatives individuelles et les énergies qui sont indispensables à l'épanouissement de l'entreprse privée.

La Chambre voit dans l'éducation économique un facteur prépondérant de l'essor et du progrès du régime d'entreprise libre au Canada, et, par conséquent, s'engage à faire tout son possible pour éclairer les particuliers et les encourager à décider d'eux-mêmes et à prendre leurs propres responsabilités. 
La Chambre reconnait qu'il incombe à la société de venir en aide aux individus incapables de se subvenir à eux-mêmes, mais elle ne croit pas qu'il soit du devoir de l'Etat de dispenser des services que les citoyens sont en mesure de se procurer. La sécurité sociale ne doit pas devenir une fin en soi. Il faut que les Canadiens se gardent de se reposer sur l'Etat à un tel point que l'initiative individuelle se trouverait émoussée.

Si l'on en vient à se reposer sur le gouvernement pour aplanir toutes les difficultés, le sens de l'initiative risque de se perdre. La Chambre préfère placer sa confiance dans l'esprit d'initiative, le plein exercice des libertés individuelles, et la ressource des citoyens. C'est sur ces derniers qu'elle compte pour assurer au peuple du Canada les meilleures conditions de vie.

\section{Recommandations}

La Chambre recommande:

1. à tous ses membres de répandre une meilleure appréciation du régime économique de liberté d'entreprise;

2. à ses Chambres membres de former des Comités des Affaires publiques, afin de stimuler l'intérêt de leurs membres à l'égard des affaires publiques à tous les paliers.

\section{Relations publiques des entreprises}

La Chambre de Commerce du Canada est d'avis que les relations entre les entreprises commerciales et le public doivent être harmonieuses si l'on veut assurer le progrès de l'économie et sauvegarder la liberté de concurrence.

Il faut pour cela que les principes directeurs des entreprises s'appuient sur la volonté de bien servir la clientèle, d'être loyaux envers les employés, les actionnaires et les fournisseurs, sans perdre de vue l'intérêt général.

Les hommes d'affaires ne devraient jamais hésiter à se prononcer sur toutes les questions d'intérêt public. Il importe que le public voie les choses dans leur perspective véritable et apprécie à sa juste valeur l'apport de l'industrie et du commerce à l'allant de notre économie.

\section{Recommandation}

Que tous ses membres tirent pleinement parti des moyens d'information disponibles, dans le but de faire apprécier le rôle de la communauté d'affaires dans l'économie, et exposent leurs vues sur les questions d'intérêt public.

\section{Communisme}

La Chambre signale qu'il est du devoir de chacun de bien saisir les dangers du communisme et la nécessité d'une constante vigilance afin de le dénoncer. Ce n'est que par les efforts individuels et collectifs de tous les vrais Canadiens que le danger peut être écarté. La défense la plus efficace contre le communisme réside 
dans la compréhension et l'appréciation entière des avantages de notre régime économique fondé sur la liberté d'entreprise, et lattachement aux principes de la démocratie.

\section{Recommandations}

1. Que tous les Canadiens apprennent à mieux connaître les principes de la démocratie, afin de la mieux apprécier;

2. Que les Chambres de commerce, bien pénétrées des dangers que représente la doctrine communiste, soient constamment sur la brèche pour la réfuter;

3. La Chambre demande aux Chambres provinciales et locales de prendre les mesures suivantes, en vue de combattre le communisme:

(a) Presser les autorités locales et provinciales en matière d'instruction publique, d'intensifier l'enseignement de la liberté d'entreprise dans les écoles;

(b) Solliciter le concours de la radio, de la TV, de la presse, du cinéma, pour répandre une meilleture connaissance de notre mode de vie;

(c) Inviter les conférenciers, orateurs, à promouvoir l'entreprise libre;

(d) Inviter leurs membres à promouvoir parmi leurs employés une meilleure appréciation des avantages de la liberté d'entreprise.

\section{Profits}

La Chambre de Commerce du Canada estime que les bénéfices commerciaux et la crainte de subir des déficits, constituent un stimulant essentiel à la bonne marche des affaires et favorise l'expansion économique. Ce régime doit être défendu dans l'intérêt du consommateur, du salarié, $d u$ fournisseur et de l'économie dans son ensemble.

L'entreprise privée est constamment sujette à la concurrence et doit veiller sans relâche à ce que ses opérations s'effectuent de la manière la plus efficace et la plus économique. Elle ne doit pas perdre de vue que toute carence d'efficacité, si on n'y remédie, amènera des pertes financières et, éventuellement, la banqueroute. C'est parce que les entreprises ne réalisent pas toujours de profits que le système est souvent désigné sous le nom de «pertes et profits ». Les bénéfices réalisés sont l'indice de l'état de santé d'une entreprise.

Il importe que le public comprenne mieux la nature et la nécessité des bénéfices commerciaux. Les fonds placés dans l'entreprise privée sont investis dans l'espoir de réaliser des profits, et de tels placements sont indispensables au maintien et à l'essor de notre économie. Les bénéfices commerciaux ne font pas que rémunérer les placements, ils sont essentiels au progrès commercial et à l'expansion économique. Ils assurent en outre un certain degré de sécurité qui protège le salarié, le consommateur, le fournisseur et l'économie dans son ensemble.

Les profits après l'acquittement des taxes ne représentent qu'un petit résidu de l'opération commerciale. La promesse de bénéfices est une invite aux investissements et leur emploi contribue à l'essor de notre économie. La prospérité d'une 
entreprise signifie plus d'emplois et de richesses, sous forme de biens et de services, et un plus haut niveau de vie pour tous.

\section{Recommandations}

1. Que les membres corporatifs s'appliquent à éclairer les employés sur la nécessité et le rôle des bénéfices dans notre régime économique;

2. Que les Chambres de commerce membres incluent ce sujet au programme de leur Comité des Affaires publiques.

Droit administratif

La Chambre de Commerce du Canada est opposée d̀ ce que le gouvernemenl, ou ses délégués, empiètent sur la liberté individuelle et celle de nos institutions nationales, en exerçant ses pouvoirs. Le droit d'appel aux tribunaux est un principe fondamental de la démocratie.

La Chambre réitère sa conviction que l'exercice de pouvoirs arbitraires par le gouvernement menace la liberté des particuliers et celle de nos institutions nationales. Lorsque le gouvernement fait délégation de ses pouvoirs législatifs, administratifs ou juridiques, la Chambre est d'avis que cette transmission devrait être limitée au bon fonctionnement de l'Etat. L'accès aux tribunaux ne devrait jamais être refusé.

\section{Recommandation}

Que ses membres soient toujours en alerte et que, lorsqu'il y a violation du principe de sauvegarde de la liberté individuelle, ils signalent le cas à la Chambre nationale ou à la Chambre provinciale, qui entreprendront les démarches qui s'imposent.

\section{Concurrence gouvernementale}

Le gouvernement ne devrait pas concurrencer l'entreprise privée dans les domaines où celle-cr suffit, ou suffrait, à assurer les services à la satisfaction générale.

La Chambre de Commerce du Canada croit que l'Etat ne devrait pas se lancer dans des activités commerciąles où l'entreprise privée opère déjà, ou pourrait le faire, à la satisfaction générale. Sous un régime de libre concurrence on ne produit que des biens ou services que l'on est sûr de pouvoir écouler. La Chambre de Commerce du Canada craint que dans les secteurs où l'Etat concurrence l'entreprise privée, les sociétés étatisées soient subventionnées à même les revenus du gouvernement. Le contribuable ne réalise pas toujours qu'une part des impôts qu'il paie sert à concurrencer l'entreprise privée.

\section{Recommandations}

L'Auditeur général du Canada devrait vérifier et rendre publics les coûts d'opération des entreprises étatisées qui font concurrence aux sociétés privées. 
Il y a de nombreux secteurs où le gouvernement livre concurrence d l'entreprise privée, et, à ce propos, la Chambre fait les recommandations spécifiques suivantes:

1. Les sociétés aériennes privées devraient jouir d'un traitement égal d celui accordé aux entreprises d'Etat;

2. Les postes privés de radio et télévision devraient jouir des mêmes avantages que les postes d'Etat;

3. Le gouvernement devrait cesser la vente d'annuités et l'opération des caisses d'épargne postales;

4. La Chambre est d'avis que la Loi sur l'Office National du Film devrait être amendée de sorte que les commandes du gouvernement canadien et des Corporations de la Couronne soient confiées à des entreprises privées. La Chambre est d'avis que le rôle de l'Office National du Film devrait se limiter à distribuer des films et à conseiller les divers services gouvernementaux et les Corporations de la Couronne. Que toutes ses activités soient restreintes et que tout soit mis en oeuvre en vue de diminuer les dépenses sous ce chef;

5. Sauf pour l'impression des documents d l'usage du Parlement, le gouvernement devrait confier l'exécution des autres publications à l'entreprise privée.

Liberté des marchés

La Chambre estime que tous les producteurs devraient jouir d'une liberté totale dans le choix des debouchés, et que la participation aux opérations des organismes établis dans le but de faciliter l'écoulement des produits devrait être facultative.

Participation oux Affaires de l'Etat

La Chambre de Commerce du Canada voudrait que tous les citoyens du pays soient bien instruits des principes de base des divers groupements et des rouages de l'Etat. La Chambre est d'opinion que tous les hommes d'affaires et, en vérité, tous les Canadiens, devraient s'intéresser au choix, à la mise en nomination, à l'élection de tous les candidats aux fonctions publiques à tous les paliers administratifs. Ils devraient de plus s'intéresser aux activités des candidats élus.

\section{Recommandation}

Les Chambres de commerce sont invitées à encourager tous leurs membres d prendre une part plus active aux affaires publiques à tous les paliers.

\section{RELATIONS PATRONALES-OUVRIÈrES}

Notre avenir économique dépend de notre habileté d conserver et étendre nos marchés domestiques et étrangers. Pour faire face à la concurrence il nous faut perfectionner sans cesse nos moyens de production. La Chambre de Commerce du Canada croit que la clef d'une telle amélioration de la production réside dans une coopération étroite fondée sur la monfiance mutuelle entre employés et patrons.

Ils devraient s'efforcer de résoudre leurs problèmes communs dans un climat de liberté. La Chambre croit que dans les cas où les employés décident de conclure 
une entente collective avec leurs employeurs, par l'intermédiaire d'un agent négociateur, le syndicat peut être un appoint à l'économie d'entreprise libre et, par voie de conséquence, contribuer au bien-être des autres citoyens en améliorant le sort des travailleurs.

La Chambre est d'opinion que toute législation devrait viser à sauvegarder la liberté individueile, tout en protégeant l'intérêt de la collectivité. Quand la loi accorde des droits aux syndicats de travailleurs, elle devrait établir les responsabilités qui y correspondent. La Chambre est d'avis que les deux groupes devraient être iusticiables au même degré.

La Chambre estime que les salaires devraient être en fonction de la productivité et qu'ils ne doivent pas augmenter s'il n'y a pas accroissement parallèle de cette dernière. Les salaires reflètent les conditions économiques, l'activité commerciale et d'autres facteurs connexes, et une ascension en spirale des salaires qui n'en tiendrait pas compte compromettrait la santé économique de la nation.

Les décisions de la Commission des relations ouvrières et les conseils d'arbitrage ont une portée juridique étendue qui touche les droits et les responsabilités financières des parties soumises à leur juridiction. La Chambre est par conséquent d'opinion qu'il devrait être permis d'en appeler en justice de leurs décisions.

C'est un principe fondamental de la démocratie que personne ne doit être tenu de révéler ses allégeances politiques. En principe, la Chambre condamne les déductions de cotisation syndicales destinées à la caisse d'un parti politique. Elle y voit une violation du droit civique et démocratique qui appartient à chaque citoyen d'appuyer le parti de son choix, sans être tenue de révéler ses préférences, et elle considère que c'est abuser des procédés de négociations collectives.

La reconnaissance syndicale accorde des pouvoirs et des droits graves de conséquences. La Chambre croit que tout doute qui pourrait exister dans l'esprit, soit des employeurs soit des employés, devrait être élucidé par le truchement d'un scrutin secret qui assure un moyen démocratique d'en arriver à une décision.

La Chambre est d'opinion qu'il n'est pas bon que le consentement du ministre soit requis de pouvoir intenter. Il appartient aux tribunaux de décider si une poursuite est fondée ou non, c'est-à-dire s'il $y$ a ou non matière à poursuite.

La Chambre est d'avis qu'il est impérieux que les juges continuent d'être éligibles à la présidence des tribunaux d'arbitrage et qu'il est souhaitable qu'ils soient éligibles à la présidence des tribunaux de conciliation.

\section{Recommandations}

La Chambre affirme qu'il est d'intérêt public :

1. Que les ententes en foi desquelles un employé est congédié, soit parce qu'il n'appartient pas ou cesse d'appartenir à un syndicat quelconque, ou qu'il n'a pas payé sa cotisation à un syndicat auquel il n'est pas affilié, soient prohibées;

2. Que des clauses de la loi soient modifiées ou élucidées de façon d assurer que les syndicats ouvriers soient justiciables; 
3. Que les grèves ou interdictions des accès aux lieux de travail (lock-out) soient interdites lorsqu'elles mettent en cause la santé publique ou la sécurité, et que les questions non réglées soient confiées pour règlement d̀ larbitrage;

4. Qu'un état de grève légale ne puisse être déclaré plus de 30 jours après que la majorité des employés en cause, sur les lieux mêmes du litige, ont voté en faveur d'une grève par scrutin secret surveillé par un tiers;

亡. Que pendant la durée d'une grève le ministre compétent ait le pouvoir d'ordonner de son propre chef, ou d la demande des parties en cause, un vote secret sur le règlement du litige et surveillé par une tierce partie;

6. Que tout piquetage soit interdit :

(a) Sauf sur les lieux mèmes où une grève ou un lock-out légaux sévissent et, dans ce cas, que le piquetage soit fait par les employés de l'établissement où la grève ou lock-out ont lieu;

(b) Quand l'objet en est la revendication de droits de négociation;

(c) Dans le cas d'un litige de juridiction;

(d) Quand l'employeur n'est pas impliqué dans le différend;

7. Que les grèves de solidarité soient prohibées;

8. Que la loi définisse clairement le boycottage secondaire et le prohibe;

9. Qu'il soit permis d'en appeler devant les tribunaux d'une décision de la Commission de la Commission des relations ouvrières et des conseils d'arbitrage, touchant un point spécifique de loi ou de juridiction;

10. Qu'après s'être assuré qu'un nombre représentatif des employés intéressés à la procédure de certification sont des membres en règle du syndicat, la Commission des relations ouvrières ordonne un vote secret sur la représentation;

11. Que l'article 46 de la loi des Relations industrielles et des différends, lequel exige le consentement du Ministère avant d'entamer toute procédure, soit rescinde ;

12. Que les juges continuent d'être éligibles d̀ la présidence des tribunaux d'arbitrage et de conciliation;

13. Que les employés occupant des postes de confiance, tels que gardiens, surveillants, etc., soient exclus de tout groupe admis d participer aux négociations.

Il est de plus recommandé que les Chambres de commerce locales encouragent le public à s'intéresser aux problèmes du Travail, et en particulier, veillent à ce que les lois afférentes au piquetage et à l'intimidation soient respectées et appliquées par les autorités compétentes.

\section{Santé nationale et SeRvices de Santé}

La Chambre estime que l'une des préoccupations premières du peuple canadien devrait être de chercher d préserver la santé nationale et, à cette fin, le concours des particuliers est d'une importance vitale. 
Le problème de la santé nationale doit être envisagé sous trois chefs : (1) la prévention; (2) la guérison et la convalescence; (3) le soin des malades chroniques et des incurables. Avant tout vient la prévention des maladies et des accidents. Cet aspect du problème doit faire l'objet d'un programme d'éducation publique en matière de santé et de sécurité, établi par les autorités fédérales et provinciales.

Il faut s'efforcer de créer au Canada des services médicaux en nombre suffisant et de former un personnel médical plus nombreux, afin de pouvoir assurer les soins médicaux et les services hospitaliers nécessaires.

La santé nationale ne dépend pas uniquement des services de santé mais aussi d'une alimentation rationnelle et d'une habitation salubre. Le programme de sécurité sociale actuel qui comprend les pensions de vieillesse, les allocations familiales, les secours aux vieillards, représentent des charges considérables et croissantes pour les Canadiens, mais contribuent à fournir des habitations salubres et une nourriture convenable. On devrait examiner rigoureusement les débours occasionnés par le présent programme de sécurité sociale.

La Chambre croit que dans une société libre c'est d'abord à l'individu qu'incombe la responsabilité de prévoir et de payer les frais médicaux de sa famille. La Chambre est d'avis que chacun devrait faire une part aux frais médicaux dans l'établissement de son budget. Elle est impressionnée par le développement rapide et la vulgarisation des plans de services et d'indemnités volontaires pré-payés. Ces plans méritent d'être encouragés et les employeurs devraient être invités à y contribuer lorsque cela est possible.

La Chambre s'inquiète de l'avenir. Elle est certaine qu'il y aura des demandes pour que les bénéfices et les avantages auxquels donnent droit les plans d'hospitalisation existants cuu gouvernement soient augmentés de façon à couvrir d'autres dépenses médicales ; tout cela requérant un accroissement des débours et représentant des frais supplémentaires pour les contribuables canadiens. Il est donc possible qu'on en vienne à un système d'assurance obligatoire et, même; à unt forme d'étatisation de la médecine.

\section{Recommandations}

1. Que toute aide future de la part du gouvernement fédéral ne devrait être offerte que dans des cas où les individus sont incapables d'assumer les frais causés par la maladie;

2. Que les Chambres de commerce provinciales présentent des recommandations aux divers gouvernements provinciaux, toucchant la recommandation No 1 qui précède.

\section{LE PLEIN EMPLO'}

L'expérience a prouvé que pour assurer le plein emploi et pour tirer pleinement parti des ressources de la nation en connaissances techniques et en capitaux, il faut une industrie manufacturière en plein essor.

Les industries manufacturières doivent être prospères, diversifiées et hautement évoluées, si l'on veut que le pays bénéficie commercialement des nouveaux 
procédés de fabrication et des progrès technologiques résultant des recherches scientifiques. Une bonne part des progrès réalisés dans la productivité et qui se sont traduits par des augmentations de revenus, ou bien encore par des prix moins élevés, sont le fait des progrès technologiques réalisés depuis le début du siècle.

Une industrie répondant aux exigences que nous avons citées plus haut non seulement offrirait un nombre plus grand d'emplois, mais créerait aussi le pouvoir d'achat nécessaire pour assurer un débouché à d'autres industries, ce qui signifierait une économie nationale plus stable, l'indépendance économique et une conjoncture moins influencée par les conditions du commerce avec les pays étrangers.

L'industrie manufacturière du Canada emploie un million cing cent mille travailleurs et les salaires payés forment une bonne part du pouvoir d'achat global. Au cours des dix dernières années tous les nouveaux emplois enregistrés dans des domaines autres que ceux des services, se situent pour ainsi dire dans les industries manufacturières. Les gens employés dans les usines offrent un vaste débouché pour les produits des industries primaires.

\section{Recommandation}

Que le gouvernement attache plus d'importance au développement des industries secondaires et prenne des mesures visant à l'accélérer.

\section{Bien-être social}

La Chambre est en faveur d'un programme de sécurité sociale à la mesure de nos moyens qui ne soit pas attentatoire au régime de liberté d'entreprise, source de revenus qui permettent les prestations et les dépenses à caractère social. Il lui semble, toutefois, qu'il serait peu sage de prendre d'autres engagements tant que l'économie n'aura pas retrouvé son allant. A l'heure présente, il existe un danger que toute nouvelle charge résultant de services supplémentaires aurait pour effet de retarder le progrès.

\section{PRINCIPES D'ECONOMIE POLITIQUE}

par

Roger Dehem

Ce manuel, à la fois élémentaire et approfondi, s'adresse principalement aux étudiants intéressés aux grands problèmes économiques de notre temps. Il propose une vue synthétique, dépouillée de tout artifice technique, de l'économ:e contemporaine. Il vise à polariser la réflexion sur les problèmes institutionnels et politiques, présentés dans une perspective historique.

1 volume, 200 pages 